\title{
EL MODELO MIXTO (PRESENCIAL-VIRTUAL) EN LA ENSEÑANZA DE LA BIOLOGÍA PARA INGENIEROS TÉCNICOS AGRÍCOLAS. UN AVANCE SOBRE LAS IMPRESIONES DE LOS ALUMNOS
}

\author{
Encarnación Núñez Olivera \\ Javier Martínez Abaigar \\ Rosario García Gómez \\ Rafael Tomás Las Heras \\ Universidad de La Rioja
}

RESUMEN: A través de una encuesta, se han analizado las impresiones de los alumnos sobre la implantación del Modelo Mixto (presencial-virtual) en la asignatura de Biología durante el curso 2002-2003. La Biología corresponde al primer curso de Ingeniería Técnica Agrícola (especialidades de Hortofruticultura y Jardinería, e Industrias Agrarias y Alimentarias) de la Universidad de La Rioja. La participación de los alumnos fue relativamente baja, ya que sólo un $30 \%$ de los matriculados rellenaron la encuesta. Los alumnos consideraron que la asignatura es útil para su formación y acogieron favorablemente la implantación del Modelo Mixto, pero valoraron peor la parte virtual que la presencial. Esto pudo ser debido a: 1) la escasa disponibilidad y conocimiento de los medios informáticos por parte de los alumnos; 2) el apego de los alumnos a métodos docentes tradicionales; 3) la mayor necesidad de apoyo profesoral que necesitan los alumnos en el primer curso universitario, y 4) los posibles fallos cometidos por los profesores en el primer curso de implantación. Se apuntan algunas sugerencias metodológicas que podrían mejorar el desarrollo del Modelo Mixto en la asignatura de Biología. Las conclusiones de este trabajo deberían ser corroboradas en el futuro mediante estudios plurianuales.

ABSTRACT: We analyzed, by means of a survey, the impressions of the students of Biology on the introduction of the Mixed Modality (presential-virtual) during the course 2002-2003. Biology is a subject belonging to the first course of Agricultural Technical Engineering (Horticulture-Fruticulture and Gardening, and Food and Agriculture Industries) at the University of La Rioja. The participation of the students was relatively low, since only $30 \%$ of the registered students answered the survey. The students considered that Biology is a useful subject for their education and they received favorably the intro- 
duction of the Mixed Modality. However, they rated lower the virtual part than the presential one. This could be due to: 1 ) the low availability of computers and scarce knowledge of informatics among the students; 2 ) the preference of the students to traditional teaching methods; 3 ) the higher professorial support needed by the students in their first university course, and 4) the possible shortcomings made by the teachers in the first course of Mixed Modality. Some methodological suggestions are outlined for the improvement in the development of the Mixed Modality in the subject of Biology. Our conclusions should be corroborated in the future by means of pluriannual studies.

PALABRAS CLAVE: Biología, docencia, Ingeniería Técnica Agrícola, Modelo Mixto, enseñanza virtual, enseñanza presencial, nuevas tecnologías.

KEYWORDS: Biology, teaching, Agricultural Technical Engineering, Mixed Modality, virtual teaching, presential teaching, new technologies.

\section{INTRODUCCIÓN}

En la Universidad de La Rioja se imparten dos especialidades de la titulación de Ingeniería Técnica Agrícola: Hortofruticultura y Jardinería (en adelante HJ) e Industrias Agrarias y Alimentarias (en adelante IAA). Los planes de estudios vigentes de ambas especialidades fueron establecidos por Resolución de la Universidad de La Rioja de 20 de abril de 1993 (B.O.E. de 2 de junio para HJ y de 9 de junio para IAA). La asignatura de Biología que se incluye en dichos planes corresponde al descriptor que aparece dentro de la materia troncal "Ciencias del Medio Natural" de las Directrices Generales Propias de las titulaciones de Ingeniería Técnica Agrícola en IAA y en HJ (R.D. 1452/1990 y 1454/1990 de 26 de octubre, B.O.E. de 20 de noviembre). En los planes de estudios de la Universidad de La Rioja, la Biología se imparte en el primer cuatrimestre del primer curso, con una carga docente de 7.5 créditos (4.5 teóricos y 3 prácticos). La asignatura tiene carácter básico, con una finalidad claramente introductoria a los conceptos científicos relacionados con los seres vivos.

Cuando el alumno inicia una carrera universitaria, procedente por tanto del Bachillerato o de los módulos de Formación Profesional, debe adaptarse a los nuevos modos de enseñanza-aprendizaje de la Universidad. Por su situación en el plan de estudios, uno de los primeros contactos del alumno con la enseñanza universitaria se tiene a través de la Biología. En consecuencia, el diseño docente de esta asignatura ha de tener en cuenta que el cambio del alumno hacia una mayor autonomía personal en el aprendizaje deberá ser gradual.

Cada día más la enseñanza universitaria tendrá que responder a situaciones de enseñanza-aprendizaje diversas, que abarcan desde situaciones convencionales hasta la enseñanza no presencial. Para lograr un aprendizaje eficaz, necesitaremos desarrollar en nuestros alumnos algunas de las capacidades implicadas en el aprendizaje abierto: la habilidad de diagnosticar las propias necesidades, de programar planes para lograr los propios objetivos, de evaluar la efectividad de las actividades de aprendizaje. Se requiere, por tanto, introducir en la enseñanza universitaria otro estilo caracterizado por potenciar en los alumnos el aprender a aprender, el aplicar el aprendizaje al mundo real, y aquí, por su adaptabilidad y modularidad, encaja perfectamente la enseñanza multimedia (Salinas Ibáñez 1994). 
Como consecuencia de lo anterior, creímos conveniente introducir cambios en la docencia tradicional de la asignatura. Ésta se ha diseñado como un sistema mixto de enseñanza presencial y enseñanza con materiales multimedia a través de la plataforma virtual WebCT. Este sistema se denomina Modelo Mixto en la Universidad de La Rioja. El material didáctico multimedia se ha concretado en:

- El bloque temático de Citología. El alumno tiene a su disposición de forma constante el material gráfico y las explicaciones necesarias para integrar la morfología celular con la fisiología, que se imparte posteriormente.

- Varias prácticas de microscopía. El estudiante dispone, en formato multimedia, de fotografías de cortes histológicos (raíz, tallo, hoja) y de las distintas fases de la mitosis de la célula vegetal, dotadas cada una de carteles y leyendas explicativas. Paralelamente el estudiante dispone de las correspondientes preparaciones para observarlas al microscopio en el laboratorio.

- Trabajo experimental dirigido (trabajo de prácticas). Este trabajo tenía carácter mixto, puesto que los alumnos, en grupos de 2 ó 3, debían seguir el ciclo completo de desarrollo de una planta tomando medidas de manera presencial, pero elaborando los resultados de acuerdo con las instrucciones detalladas de forma virtual. Los resultados y las conclusiones se debían presentar por escrito de acuerdo con el formato explicativo incluido en la plataforma. Así mismo, los alumnos tenían, como apoyo virtual, fotografías explicativas de las distintas fases de desarrollo de la planta.

- En las prácticas presenciales de laboratorio, todos los guiones estaban en formato multimedia en la plataforma, de forma que los alumnos debían leerlos antes del inicio de la correspondiente práctica.

En la elaboración de los materiales se tuvieron en cuenta los criterios de Insa y Morata (1998): que estén integrados en un todo coherente, que den información al usuario en tiempo real, y que permitan la interactividad entre el usuario y el programa. Entendemos que los contenidos se presentan en una secuenciación y estructuración adecuada, de forma atrayente, de modo que se motive al estudiante. El volumen de información presentado es suficiente para el dominio correcto de los contenidos por parte del estudiante y la adquisición de los objetivos previstos en el programa.

Respecto a las consideraciones estéticas que deben de contemplarse en el diseño de pantalla, se ha tenido en cuenta el equilibrio entre texto e imágenes, la utilización de la negrilla o cursiva como elementos para la llamada de atención, el volumen de información situada en la pantalla, el espaciado interlineal, etc., ya que parece ser que repercuten directamente sobre la comprensión de la información presentada (Cabero Almenara y Duarte Hueros 1999). El diseño de pantallas facilita la navegación abierta por los contenidos, mediante la unificación perceptiva de todos los elementos. El estudiante en todo momento puede identificar dónde se encuentra, qué recorrido está realizando, y desde dónde puede volver a incorporarse a una posición determinada de los contenidos.

La Universidad de La Rioja está interesada en que la enseñanza multimedia se extienda a todas las titulaciones, de modo que sus estudiantes adquieran destreza en el uso de esta nueva herramienta, pilar de la sociedad de la información. En consecuencia, ha potenciado la conversión de asignaturas tradicionalmente presenciales al 
Modelo Mixto presencial-virtual. En la actualidad, alrededor de 40 asignaturas de diversas titulaciones se imparten dentro de esta modalidad. Por otro lado, la necesaria convergencia universitaria con Europa y la progresiva adaptación al sistema de créditos europeos, obliga a los profesores a introducir nuevos modos de enseñar. Cada profesor intenta que sus alumnos se esfuercen por aprender y no sólo por aprobar (Alonso Tapia 2001), y a tal fin pueden renovar cada curso no sólo los contenidos sino las actividades de aprendizaje. En estos principios se enmarca el trabajo que se presenta.

Entendemos que una parte del éxito de la enseñanza reside en el modo en que los alumnos aprenden y en su aceptación del método de enseñanza propuesto. Por ello, el objetivo del presente trabajo es conocer el grado de satisfacción de los estudiantes respecto del modo en que se ha impartido la asignatura de Biología en las dos especialidades de Ingeniería Técnica Agrícola (HJ e IAA) en el curso académico 2002-2003, con especial referencia a esta nueva modalidad de enseñanza-aprendizaje, el Modelo Mixto, que mezcla la enseñanza presencial tradicional con la enseñanza a través de la red. Aunque en España se está avanzando mucho en los últimos años en la aplicación de los entornos virtuales a la enseñanza (Gisbert, Adell, Anaya y Rallo 1997; Sancho 1998; GET 1998), no conocemos ningún estudio previo sobre la valoración del Modelo Mixto por parte de los alumnos en asignaturas o titulaciones similares a las consideradas en nuestro estudio.

\section{MATERIAL Y MÉTODOS}

Para conocer el grado de satisfacción de los alumnos sobre la implantación del Modelo Mixto (presencial-virtual) en la asignatura de Biología (primer curso de las Ingenierías Técnicas Agrícolas), se elaboró una encuesta de 17 items (ver ANEXO), que fue cumplimentada al finalizar el cuatrimestre y sin haber realizado la prueba del examen. El cuestionario trata de incorporar un conjunto de variables que subyacen en el proceso de enseñanza, como son las que tienen relación en primer lugar con el propio sujeto (ser repetidor o no, preferencias hacia el modo de aprender, percepción del proceso educativo) pero también con el proceso didáctico (recursos didácticos, en especial los recursos multimedia, actividades de aprendizaje diseñadas, contenidos del programa, etc.). Interesaba asimismo analizar hasta que punto la aceptación o rechazo de la asignatura iban ligados al diseño y desarrollo de la misma o al propio profesor.

Las variables y dimensiones que se han querido analizar son :

- situación del alumno en calidad de repetidor o no, y correlación con la aceptación del modelo, ya que se trataba del primer año que se impartía la asignatura de este modo

- valoración del programa

- valoración del desarrollo de la asignatura y de las diferentes actividades llevadas a cabo, en especial las referidas a enseñanza multimedia

- valoración del sistema de evaluación propuesto

- valoración y nivel de satisfacción sobre el profesor

- percepción de la utilidad formativa de la asignatura

- detectar aspectos de interés para los alumnos y no incluidos en el programa actual. 
La encuesta se pasó a los alumnos el 21 de enero de 2003, una vez que había finalizado la docencia de la asignatura y sólo restaba su evaluación (realización de exámenes y entrega y corrección de trabajos). Los alumnos rellenaron la encuesta en horario lectivo durante el tiempo que estimaron oportuno (un máximo de 20 minutos). De los 70 alumnos matriculados en $\mathrm{HJ}$ y los 85 en IAA, se obtuvieron las siguientes respuestas: en $\mathrm{HJ}, 10$ de alumnos nuevos y 16 de repetidores; en IAA, 13 y 7 , respectivamente.

En aquellas preguntas de la encuesta cuya respuesta era numérica, se han realizado ANOVAs de una vía para comprobar los efectos de la especialidad $(\mathrm{H})$ frente a IAA) y del tipo de alumno (repetidores frente a alumnos de nueva matriculación), una vez comprobadas la normalidad y homoscedasticidad de los datos. También se han hecho análisis de t de Student por parejas para comprobar la significación de las diferencias entre las medias de las distintas variables. Los procedimientos estadísticos se ejecutaron con el programa SPSS 10.0 para Windows.

\section{RESULTADOS Y DISCUSIÓN}

\subsection{Número de encuestas realizadas}

Se obtuvieron 46 respuestas, un 30\% de los alumnos matriculados. Este bajo porcentaje de participación puede ser reflejo de varios hechos: 1) la baja asistencia a clase, un fenómeno generalizado en el primer curso de estas titulaciones; 2) la proximidad del periodo de exámenes, que pudo potenciar el absentismo.

\subsection{Valoración de los distintos aspectos de la asignatura (preguntas 2 y 3)}

En la Figura 1 se representa la valoración de los distintos aspectos de la asignatura (preguntas 2 y 3) por parte de los alumnos de ambas especialidades en conjunto. Esta valoración variaba entre 5.9 (prácticas virtuales) y 7.4 (labor de los profesores). Este último valor era incluso superior a la valoración global de la asignatura (7.2), aunque no había diferencias significativas entre ambas variables. La parte teórica presencial obtuvo mejores resultados que la parte teórica virtual $(P<0.01)$, y la parte práctica presencial que la práctica virtual $(\mathrm{P}<0.001)$. Estos resultados están de acuerdo con los encontrados por Navaridas Nalda $(2001,2002)$ en un estudio sobre los modos de aprender de los estudiantes de la Universidad de La Rioja. Según este autor, el modo preferente para los alumnos es el individual, aunque las explicaciones del profesor son valoradas también de forma positiva; por el contrario, los modos menos eficaces son el estudio acompañado y la enseñanza asistida por ordenador. En este sentido, Cabero Almenara y Duarte Hueros (1999) señalan que entre las limitaciones de los estudiantes para afrontar el aprendizaje a través de la red, se encuentra la posibilidad de que los estudiantes pasen por el conocimiento de forma desmotivada e instruccionalmente inexistente, ya que el mero recorrido por las diferentes pantallas y sistemas simbólicos no significa que se produzca aprendizaje o adquisición de información.

Apenas existían diferencias significativas entre ambas especialidades en la valoración de los distintos aspectos de la asignatura (Figura 2). Sólo el trabajo de prácticas y la parte teórica virtual estuvieron mejor valorados $(\mathrm{P}<0.05)$ en una especialidad que en otra, concretamente mejor en HJ que en IAA. En ambas especialidades, la 


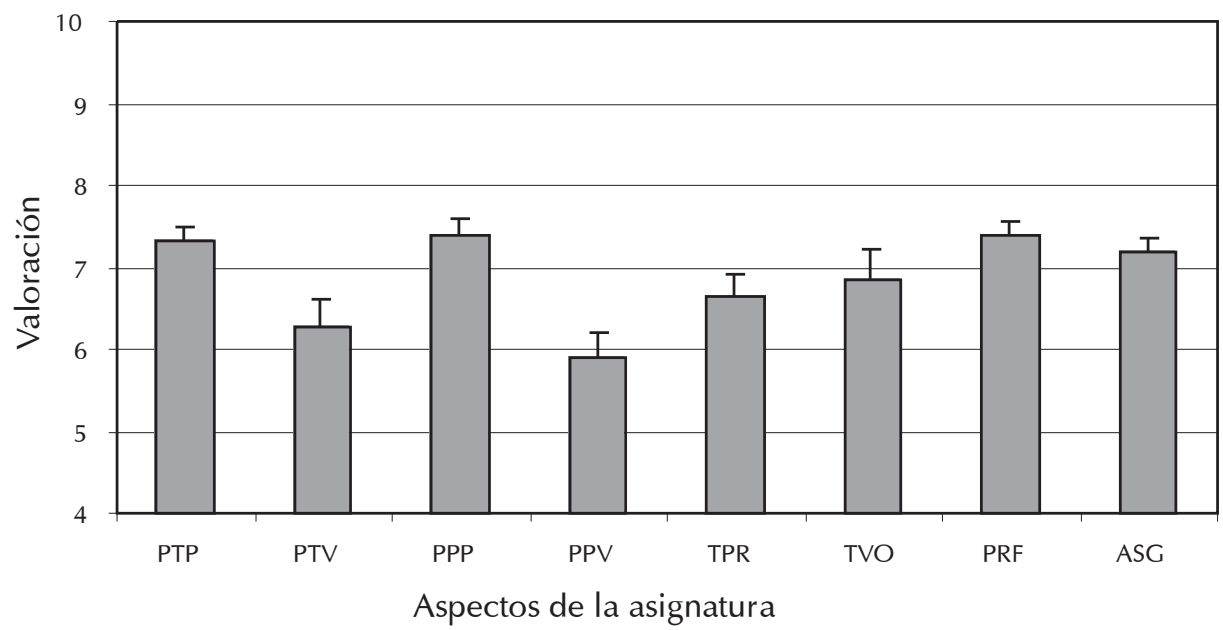

Figura 1. Valoración (media + error estándar) de los distintos aspectos de la asignatura de Biología por parte de los alumnos de ambas especialidades en conjunto de la titulación de Ingeniería Técnica Agrícola (Hortofruticultura y Jardinería, e Industrias Agrarias y Alimentarias). PTP, parte teórica presencial. PTV, parte teórica virtual. PPP, parte práctica presencial. PPV, parte práctica virtual. TPR, trabajo de prácticas. TVO, trabajo voluntario. PRF, profesorado. ASG, asignatura.

parte práctica presencial fue mejor valorada que la parte práctica virtual $(\mathrm{P}<0.01)$. También en ambas especialidades, la parte teórica presencial obtuvo mejores resultados que la parte teórica virtual, pero las diferencias fueron significativas sólo en IAA $(\mathrm{P}<0.01)$.

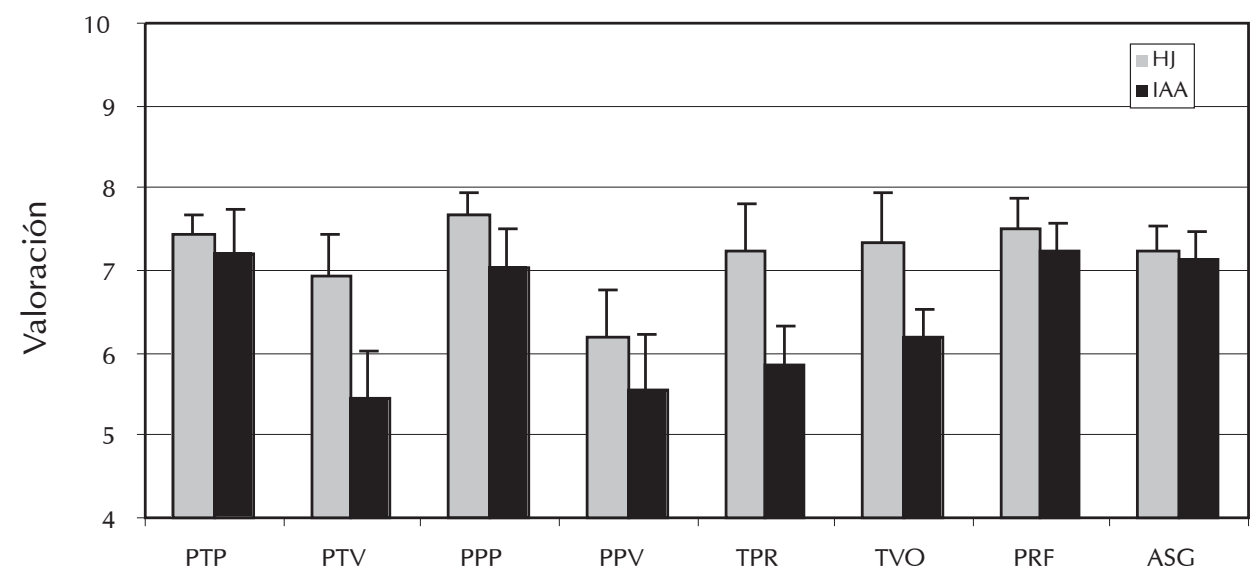

Aspectos de la asignatura

Figura 2. Valoración (media + error estándar) de los distintos aspectos de la asignatura de Biología por parte de los alumnos de las dos especialidades consideradas de la titulación de Ingeniería Técnica Agrícola: Hortofruticultura y Jardinería (HJ), e Industrias Agrarias y Alimentarias (IAA). PTP, parte teórica presencial. PTV, parte teórica virtual. PPP, parte práctica presencial. PPV, parte práctica virtual. TPR, trabajo de prácticas. TVO, trabajo voluntario. PRF, profesorado. ASG, asignatura. 


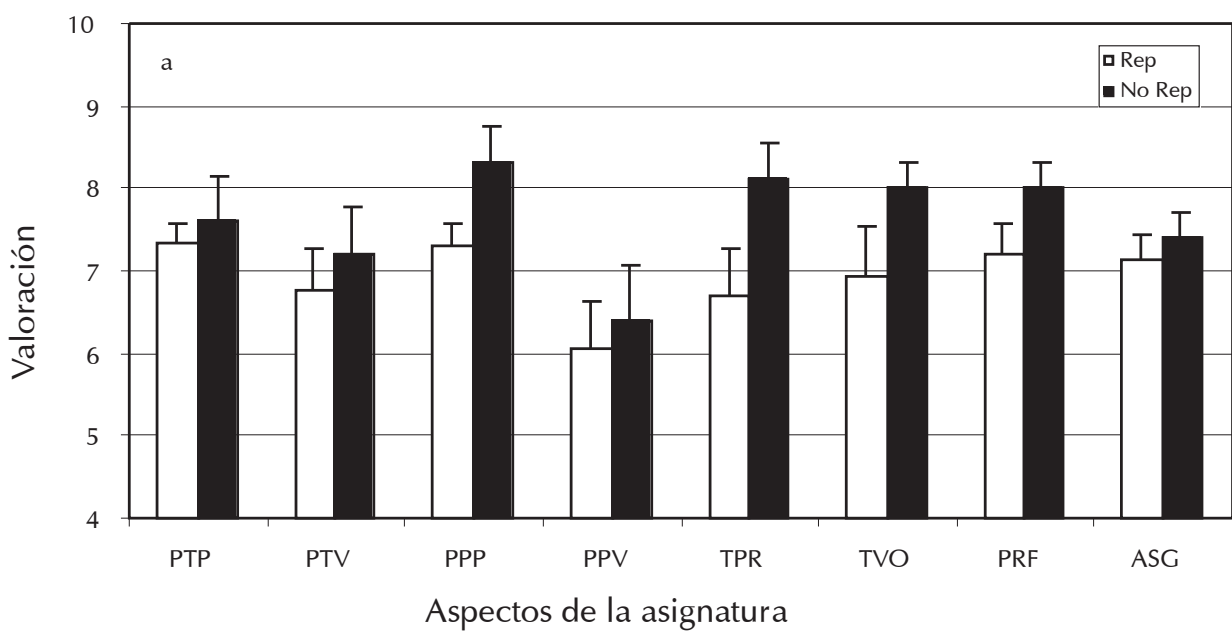

En $\mathrm{HJ}$, todos los aspectos de la asignatura fueron mejor valorados por los alumnos no repetidores que por los repetidores (Figura 3 ), aunque las diferencias sólo eran significativas en la parte práctica presencial $(\mathrm{P}<0.05)$. En IAA, las partes virtuales de la asignatura, tanto de teoría como de prácticas, así como el trabajo voluntario, fueron peor valorados por los alumnos no repetidores (aunque sólo había diferencias significativas en los dos primeros casos: $\mathrm{P}<0.01)$. Incluso, los alumnos no repetidores otorgaron calificaciones por debajo de 5 a ambos aspectos virtuales de la asignatura.

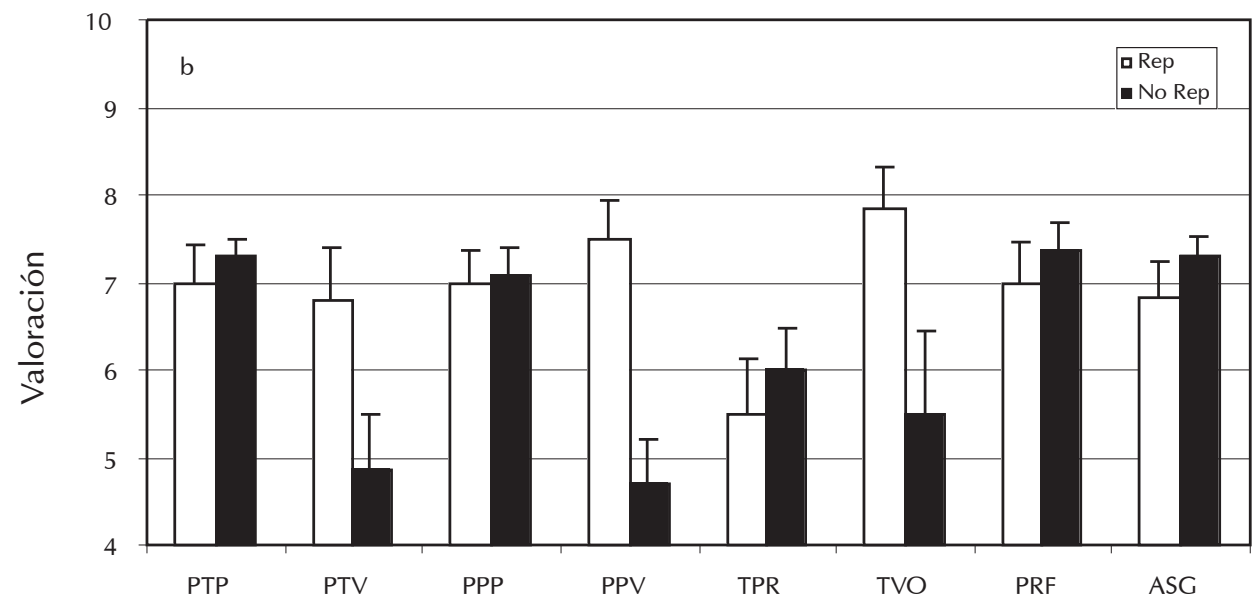

Aspectos de la asignatura

Figura 3. Valoración (media + error estándar) de los distintos aspectos de la asignatura de Biología por parte de los alumnos repetidores y no repetidores de las dos especialidades consideradas de la titulación de Ingeniería Técnica Agrícola: Hortofruticultura y Jardinería (a) e Industrias Agrarias y Alimentarias (b). PTP, parte teórica presencial. PTV, parte teórica virtual. PPP, parte práctica presencial. PPV, parte práctica virtual. TPR, trabajo de prácticas. TVO, trabajo voluntario. PRF, profesorado. ASG, asignatura. 


\subsection{Comentarios sobre el resto de las preguntas de la encuesta}

Alrededor de un $80 \%$ de los alumnos repetidores consideraron que la asignatura había mejorado con respecto a años anteriores en los que no se aplicó el Modelo Mixto. Ninguna parte concreta de la teoría gustó o disgustó especialmente a los alumnos. En la parte práctica, el "Ciclo completo de alubia" y la "Separación de pigmentos por cromatografía en capa fina" fueron las prácticas claramente más atractivas en HJ e IAA, respectivamente, mientras que no hubo ninguna práctica especialmente rechazada.

Un $60 \%$ de los alumnos opinaron que les parecía bien o muy bien que la asignatura tuviese una parte presencial y otra virtual. El 43\% de los alumnos optaba por mantener igual la carga virtual de la asignatura, mientras que el 23\% preferiría aumentarla y el $34 \%$ disminuirla.

La aceptación del profesorado revelada por la pregunta 2 se confirmó en la pregunta 11, en la que al $85 \%$ de los alumnos les gustaría que los profesores de la asignatura les impartiesen otras asignaturas en el futuro.

Los dos aspectos más generalmente reclamados por los alumnos para ser implantados en la asignatura fueron, por este orden, los exámenes parciales y las excursiones. Por el contrario, los alumnos apenas señalaron aspectos sobrantes en la asignatura.

Los criterios de evaluación fueron valorados con bien o muy bien en el $82 \%$ de los casos, y ningún alumno contestó mal o muy mal. En consonancia con estos resultados, los porcentajes asignados a las distintas actividades evaluadas fueron parecidos a los que se aplicaron por parte de los profesores. En algunos casos, los alumnos apuntaron que debería evaluarse la asistencia a clase y el interés demostrado. Para comprobar el posible efecto que pudiera haber tenido la implantación del Modelo Mixto sobre el éxito de los alumnos en la asignatura, se comparó el porcentaje de aprobados en la convocatoria de febrero de dos cursos consecutivos, 2001-2002 y 2002-2003, en ambas especialidades. En HJ apenas hubo diferencias entre ambos cursos: $16 \%$ de aprobados con respecto a los matriculados y $38 \%$ con respecto a los presentados a examen en el curso 2001-2002, y respectivamente $18 \%$ y $39 \%$ en el curso 2002-2003. Sin embargo, las diferencias fueron mucho mayores en IAA: $21 \%$ y $49 \%$ en $2001-2002$ frente a $11 \%$ y $29 \%$ en $2002-2003$.

Tan sólo un alumno consideró que la asignatura no era útil para su formación.

Entre las sugerencias expresadas por los alumnos, las más repetidas fueron que las partes virtuales se hiciesen también presenciales, especialmente en el caso de las prácticas. El principal argumento esgrimido para realizar esta sugerencia fue la carencia de ordenador. Hay que tener en cuenta que una parte de los alumnos viven en pisos o residencias en los que no existen ordenadores o conexión a Internet, por lo que dependen exclusivamente del equipamiento multimedia ofrecido por la Universidad. También apareció la sugerencia de realizar exámenes parciales y excursiones, aspectos ya detectados en otras preguntas de la encuesta.

\section{CONCLUSIONES}

1. La asignatura de Biología está bien considerada por parte de los alumnos de Ingenierías Técnicas Agrícolas, tanto de Hortofruticultura y Jardinería como de Industrias Agrarias y Alimentarias. Los alumnos opinan que es útil para su formación y la valoran globalmente con un 7.2. 
2. La implantación del Modelo Mixto ha sido bien acogida por los alumnos, especialmente por los repetidores, que consideran que la asignatura ha mejorado con este cambio. Sin embargo, los alumnos (tanto repetidores como no repetidores) valoran peor la parte virtual que la parte presencial, como ocurre en general entre los estudiantes de la Universidad de La Rioja (Navaridas Nalda 2002). En el caso de la Biología, esto puede ser debido a varios factores:

a) La escasa disponibilidad de medios informáticos por parte de los alumnos.

b) La falta de costumbre de los alumnos, en especial de los de nueva matriculación, para asimilar la aplicación de las nuevas tecnologías a la docencia. Esto viene apoyado por el hecho de que algunos métodos docentes tradicionales como las clases presenciales (teóricas y prácticas) o la elaboración de trabajos son mejor valorados que las partes virtuales.

c) La escasez de conocimientos de informática a nivel de usuario por parte de los alumnos. Resultó Ilamativo que ningún alumno utilizó las tutorías virtuales ni la posibilidad ofrecida por los profesores de enviar sus trabajos por correo electrónico.

d) La escasa autonomía de aprendizaje que tienen los alumnos universitarios de primer curso. Probablemente necesitan recibir más directrices por parte del profesor en forma presencial (por ejemplo, reclaman exámenes parciales). Esta necesidad es probablemente mayor en el caso de las asignaturas del primer cuatrimestre, como la Biología, puesto que este cuatrimestre es el primer contacto que tienen los estudiantes con la Universidad.

e) Las posibles deficiencias que los profesores hayamos podido cometer en la implantación, por primera vez, del Modelo Mixto aplicado a la asignatura de Biología.

3. La aplicación del modelo exclusivamente virtual a las partes prácticas de la asignatura parece delicado, ya que éstas suelen requerir el contacto directo con técnicas, aparatos, plantas, etc. No obstante, resulta muy interesante un apoyo virtual en aquellas prácticas con contenidos descriptivos o para ilustrar protocolos de laboratorio.

4. Como sugerencias metodológicas para mejorar la implantación del Modelo Mixto, parecen adecuadas las siguientes medidas:

a) Los profesores deberían apoyar presencialmente, o a través de ejercicios elaborados al efecto, el aprendizaje de los contenidos virtuales de la asignatura, dado que el simple hecho de implementar éstos no garantiza su aprendizaje por parte de los alumnos.

b) Se debería aumentar la formación informática de los alumnos, en especial el número de cursos para el aprendizaje de la utilización de la plataforma WebCT.

c) Se debería aumentar la disponibilidad de aulas informáticas para los alumnos, incluso en exclusiva para los alumnos del Modelo Mixto. 
5. No queda clara la influencia de la implantación del Modelo Mixto sobre el éxito de los alumnos en la asignatura. Mientras que en $\mathrm{HJ}$ apenas existieron diferencias entre los cursos 2001-2002 y 2002-2003, en IAA el porcentaje de éxito se redujo en un $20 \%$ (porcentaje de alumnos aprobados en relación a los presentados a examen en la convocatoria de febrero). En la segunda especialidad pudieron haber influido otros factores ajenos a la implantación del Modelo Mixto.

6. Resulta difícil establecer comparaciones con otros estudios similares, puesto que no conocemos intentos previos de evaluar las impresiones de los alumnos sobre la implantación de Modelos Mixtos en asignaturas o titulaciones relacionadas.

7. Dado que la aplicación del Modelo Mixto a la asignatura de Biología ha tenido lugar tan sólo durante un curso académico, tanto nuestros resultados como las conclusiones son provisionales, a falta de un estudio plurianual.

\section{AGRADECIMIENTOS}

Al Vicerrectorado de Nuevas Tecnologías y Equipamiento Informático de la Universidad de La Rioja, por la financiación de la implantación del Modelo Mixto en la asignatura de Biología.

\section{BIBLIOGRAFÍA}

ALONSO TAPIA, J. (2001). Motivación y estrategias de aprendizaje. Principios para su mejora en alumnos universitarios. En GARCÍA-VALCÁRCEL, A. Didáctica universitaria (79-111). Madrid: La Muralla.

CABERO ALMENARA, J. y DUARTE HUEROS, A. (1999). Evaluación de medios y materiales de enseñanza en soporte multimedia. Pixel-Bit. Revista de Medios y Educación, 13, 23-45.

GET (Grupo de Educación y Telemática) (1998). EVE-A: un entorno virtual de enseñanza-aprendizaje y su aplicación a la formación. En AREA, M. (ed.) Tecnologías de la Información y Educación. ¿Qué enseñamos y qué investigamos en la Universidad española? Actas de las VI Jornadas Universitarias de Tecnología Educativa. Tenerife: Universidad de La Laguna.

GISBERT, M; ADELL, J; ANAYA, L. y RALLO, R. (1997). Entornos de formación Presencial, Virtual y a Distancia. Boletín de Redlris, 40, 13-25.

INSA, D. y MORATA, R. (1998). Multimedia e Internet. Madrid: Paraninfo.

NAVARIDAS NALDA, F. (2001). Analysis of teaching strategies at the University of La Rioja: Proposal for improvement. Ann Arbor, Michigan: ProQuest Information and Learning.

NAVARIDAS NALDA, F. (2002). La actividad didáctica universitaria desde la prerspectiva de los estudiantes: un estudio de caso. En Actas del $2^{\circ}$ Congreso Internacional de Docencia Universitaria e Innovación. Tarragona: Publicaciones ICE de la Universidad de Barcelona.

SALINAS IBÁÑEZ, J. (1994). Hipertexto e hipermedia en la enseñanza universitaria. Pixel-Bit. Revista de Medios y Educación, 1, 15-29. 
SANCHO, J.M. (EQUIPO ETIC: EDUCACIÓN Y TECNOLOGÍAS DE LA INFORMACIÓN Y LA COMUNICACIÓN) (1998). Balances y propuestas sobre líneas de investigación sobre Tecnología Educativa en España: una agenda provisional. En AREA, M. (ed.), Tecnologías de la Información y Educación. ¿Qué enseñamos y qué investigamos en la Universidad española? Actas de las VI Jornadas Universitarias de Tecnología Educativa. Tenerife: Universidad de La Laguna.

\section{ANEXO I}

1. ¿Soy repetidor en esta asignatura? SI $\square$ NO $\square$

2. Valora de 0 a 10 los siguientes aspectos de la asignatura:

Parte teórica presencial $\square$

Parte teórica virtual (citología)

Prácticas presenciales

Prácticas virtuales

Trabajo de prácticas (ciclo completo de alubia) Trabajo voluntario

Labor de los profesores de la asignatura

3. Valora de 0 a 10 la opinión global que tienes sobre la asignatura

4. ¿Crees que la asignatura ha mejorado con respecto a otros años? SI NO (Contesta únicamente si eres repetidor)

5. ¿Cuál es la parte de la teoría que más te ha gustado?

6. ¿Cuál es la parte de la teoría que menos te ha gustado?

7. ¿Cuál es la práctica que más te ha gustado?

8. ¿Cuál es la práctica que menos te ha gustado?

9. Opina sobre el hecho de que que la asignatura haya tenido una parte virtual (Muy Bien/Bien/Regular/Mal/Muy Mal)

10. Valora la extensión de la parte virtual

(Debería : Aumentar/Disminuir/Quedarse igual)

11. ¿Te gustaría que los profesores de la asignatura te impartiesen otras asignaturas en el futuro? SI $\mathrm{NO}$ 
12. Indica lo que a tu parecer falta en la asignatura

$\square$ No falta nada, todo es perfecto

$\square$ Más prácticas sobre

$\square$ Más teoría sobre

$\square$ Excursiones para ver

$\square$ La realización de trabajos individuales

$\square$ Autoevaluaciones

$\square$ Exámenes parciales

$\square$ Participación de los alumnos en las clases

$\square$ Más horas de tutorías

$\square$ Más información sobre libros adecuados para prepararse la asignatura

$\square$ Más información sobre páginas de Internet para prepararse la asignatura

$\square$ Otras cosas. Especificar

13. Qué sobra en la asignatura:

$\square$ La parte presencial $\square$ La parte virtual

$\square$ Prácticas (especificar)

$\square$ Teoría (especificar)

$\square$ Los trabajos

$\square$ Otras cosas (especificar)

14. Valora los criterios de evaluación de la asignatura (Muy Bien/Bien/Regular/Mal/Muy Mal)

15. Indica qué porcentaje de la evaluación debería asignarse a cada una de las siguientes actividades:

Examen teórico

Examen práctico

Trabajo de prácticas (ciclo completo de alubia) Preguntas en prácticas

Trabajo voluntario

Otras actividades que deberían evaluarse (especificar) 
EL MODELO MIXTO (PRESENCIAL-VIRTUAL) EN LA ENSEÑANZA DE LA BIOLOGÍA PARA INGENIEROS TÉCNICOS AGRÍCOLAS. UN AVANCE SOBRE LAS IMPRESIONES DE LOS ALUMNOS

16. ¿Crees que la asignatura es útil para tu formación? SI

$\mathrm{NO}$

17. Añade algunas sugerencias para mejorar la asignatura : 